\title{
Zoledronic Acid Induces a Significant Decrease of Circulating Endothelial Cells and Circulating Endothelial Precursor Cells in the Early Prostate Cancer Neoadjuvant Setting
}

\author{
Daniele Santini $^{\mathrm{a}} \quad$ Alice Zoccoli $^{\mathrm{a}} \quad$ Chiara Gregorj $^{\mathrm{b}} \quad$ Melania Di Cerbo ${ }^{\mathrm{b}}$ \\ Michele Iuliani $^{a} \quad$ Francesco Pantano $^{a}$ Rita Zamarchi ${ }^{d}$ Federico Sergi ${ }^{c}$ \\ Gerardo Flammia $^{c}$ Maurizio Buscarini ${ }^{c}$ Sergio Rizzo ${ }^{e}$ Giuseppe Cicero ${ }^{e}$ \\ Antonio Russo $^{\text {e }}$ Bruno Vincenzi $^{\mathrm{a}}$ Giuseppe Avvisati ${ }^{\mathrm{b}}$ Giuseppe Tonini ${ }^{\mathrm{a}}$ \\ ${ }^{a}$ Medical Oncology, ${ }^{b}$ Hematology and ' Urology, University Hospital Campus Bio-Medico, Rome, ${ }^{d}$ Veneto Institute \\ of Oncology IOV- IRCCS, Padua, and 'Section of Medical Oncology, Department of Surgical, Oncological and \\ Stomatological Sciences, University of Palermo, Palermo, Italy
}

\section{Key Words}

Prostate cancer $\cdot$ Zoledronic acid $\cdot$ Circulating endothelial cells · Circulating endothelial precursor cells · Angiogenesis

\begin{abstract}
Purpose: Published data demonstrated that zoledronic acid (ZOL) exhibits antiangiogenetic effects. A promising tool for monitoring antiangiogenic therapies is the measurement of circulating endothelial cells (CECs) and circulating endothelial precursor cells (CEPs) in the peripheral blood of patients. Our aim was to investigate the effects of ZOL on levels of CECs and CEPs in localized prostate cancer. Methods: Ten consecutive patients with a histologic diagnosis of low-risk prostate adenocarcinoma were enrolled and received an intravenous infusion of ZOL at baseline (T0), 28 days (T28) and 56 days (T56). Blood samples were collected at the following times: T0 (before the first infusion of ZOL), T3 (72 h after the first dose), T28, T56 (both just before the ZOL infusion) and T84 (28 days after the last infusion of ZOL) and CEC/CEP levels were directly quantified by flow cytometry at all these time points. Results: Our analyses highlighted a significant
\end{abstract}

\begin{tabular}{ll}
\hline KARGER & ○ 2013 S. Karger AG, Basel \\
& $0030-2414 / 13 / 0856-0342 \$ 38.00 / 0$ \\
$\begin{array}{l}\text { E-Mail karger@karger.com } \\
\text { www.karger.com/ocl }\end{array}$ &
\end{tabular}

reduction of mean percentage of CECs and CEPs after initiation of ZOL treatment $[p=0.014$ (at day 3 ) and $p=0.012$ (at day 84), respectively]. Conclusion: These preliminary results demonstrate that ZOL could exert an antiangiogenic effect in early prostate cancer through CEP and CEC modulation.

๑) 2013 S. Karger AG, Basel

\section{Introduction}

Zoledronic acid (ZOL) is a third-generation bisphosphonate currently utilized in the management of bone metastases from a variety of solid tumors and in multiple myeloma. Bisphosphonates bind to hydroxyapatite bone mineral surfaces around resorbing osteoclasts and are selectively internalized by these cells inhibiting their activity [1]. ZOL inhibits the mevalonate pathway causing deregulation of GTPases signaling pathway such as Ras, Rho and Rac, ulti-

The manuscript includes a translational phase II open-label trial. EudraCT No. 2009-014446-28. 
mately leading to osteoclast apoptosis with consequent reduction in tumor cell-induced bone resorption and destruction [2]. Although the osteoclast is the main target of bisphosphonates, there are also preclinical data indicating that bisphosphonates may have effects on cells other than osteoclasts, including tumor cells. Preclinical studies on cancer cells demonstrate increased apoptosis, reduced migration, invasion, adhesion and proliferation after bisphosphonate treatment. Bisphosphonates also exhibit indirect antitumor activities by inhibiting angiogenesis and tumorassociated macrophage infiltration and promoting gammadelta T cell cytotoxicity [3]. Moreover, emerging data suggest that ZOL induces significant and long-lasting modifications in vivo in terms of reduction of circulating VEGF levels in cancer patients with bone metastases $[2,4]$. A promising area of antiangiogenic monitoring is the measurement of circulating endothelial cells (CECs) and circulating endothelial precursor cells (CEPs) in the peripheral blood of patients. CEPs, primarily derived from bone marrow and mobilized by VEGF and other angiogenic cytokines, significantly contribute to tumor angiogenesis [5, 6]. Moreover, it has been demonstrated that agents blocking VEGF signals may inhibit mobilization or integration of CECs in to tumor angiogenic vessels to impair tumor growth $[7,8]$. In healthy subjects, CECs are a very rare cell population representing 1/1,000-100,000 of circulating blood cells [9]. In many pathological conditions, the number of CECs is increased [10]. CEC and CEP levels are increased in the peripheral blood of patients affected by some types of cancer and return to normal values in patients undergoing complete remission [9]. Interestingly, Yamada et al. [11] reported that ZOL dose-dependently inhibited CEP differentiation in vitro, even at low drug levels, while at high concentrations, ZOL induced the apoptotic death of putative CEPs.

This preclinical evidence and the absence of studies on humans provide the rationale to investigate the effects of ZOL on CEC and CEP levels and its possible use as neoadjuvant therapy on localized prostate cancer. We report here the results obtained in our translational phase II study aimed at analyzing CEC and CEP modulations in 10 consecutive patients affected by localized prostate cancer and treated with ZOL.

\section{Patients and Methods}

This protocol was designed as a translational phase II openlabel trial according to the requirements of Good Clinical Practice of the European Union and the latest revision of the Helsinki Declaration. The study was approved by the Biomedical Ethics Committee of the Campus Bio-Medico, University of Rome. Patients
Table 1. Summary of the inclusion and exclusion criteria applied in the study

\section{Inclusion criteria}

- Men with histological diagnosis of localized prostate cancer

- Stage T1c or T2a disease and prostate-specific antigen value of $\leq 10 \mathrm{ng} / \mathrm{ml}$ and a Gleason score of $\leq 6$

- Men older than 75 years or a life expectancy of less than 10 years: prostate-specific antigen can be $>10 \mathrm{ng} / \mathrm{ml}$ or the Gleason score can be $7(3+4)$

- WHO performance status 0-2

- Written informed consent to all required assessments

- Patients must have undergone a core biopsy for the diagnosis of their prostate cancer

Exclusion criteria

- T0, T1a-b, T2b-c, T3 tumors and/or Gleason $>7$ and/or prostate-specific antigen $>10$ if life expectancy $>10$ years

- Previous hormonal therapy or radiation therapy for prostate cancer

- Evidence of metastatic disease or recurrent prostate cancer

- Previous diagnosis of malignancy unless nonmelanomatous skin cancer

- Serum creatinine $>1.5 \times$ upper limit of normal reference range or calculated creatinine clearance $<40 \mathrm{mls} / \mathrm{min}$

- Prior treatment with bisphosphonates in the last year

- Requiring anticoagulation with warfarin or coumarin derivatives

- Known hypersensitivity to bisphosphonates

- Current active dental problems including dental abscess or infection of the jawbone (maxilla or mandible) or a current or prior diagnosis of osteonecrosis of the jaw

- Recent (within 4 weeks) or planned dental or jaw surgery (recent dental fillings, teeth scaling, polishing or minor gingival surgery do not exclude the patient)

- History of bone metabolism diseases

- History of active acute and/or chronic inflammatory diseases

- Fever $\left(>37.5^{\circ} \mathrm{C}\right)$ in the 2 weeks before entering the study

- Concomitant prolonged treatment with steroids (either i.v. or oral)

eligible for this study were identified during the hospital prostate multi-disciplinary meeting and were then invited to participate to the study. From October 2009 to October 2011, 10 consecutive patients with a histologic diagnosis of low-risk prostate adenocarcinoma who were candidates to active surveillance before surgical procedure were enrolled. The participation in the study did not alter the standard medical and surgical therapy for prostate carcinoma. Inclusion and exclusion criteria are summarized in table 1.

All patients received an intravenous infusion of ZOL at baseline (T0), 28 days (T28) and 56 days (T56). Upon completion of the ZOL treatment, patients underwent radical prostatectomy and subsequently started a standard follow-up.

Blood samples for CEC and CEP analysis were performed at the following times: T0 (before the first infusion of ZOL), T3 (72 h after the first dose), T28, T56 (both just before the ZOL infusion) and T84 (28 days after the last infusion of ZOL), corresponding to the radical surgery. 
Flow Cytometric Analysis

Blood samples were collected in tubes containing EDTA as anticoagulant from 3 healthy subjects and 10 enrolled patients. The first $2 \mathrm{ml}$ of blood were incubated with $20 \mathrm{ml}$ of lyse solution (Pharm Lyse, BD) for $10 \mathrm{~min}$ at room temperature, and the tube was then centrifuged for $10 \mathrm{~min}$ at $1,600 \mathrm{rpm}$ at room temperature. A volume of $100 \mu \mathrm{l}$ of mononuclear cells was transferred into two FACS tubes and a multistep manual technique was used to detect and quantify CECs and CEPs.

Samples were incubated at room temperature in the dark for $15 \mathrm{~min}$ and then washed with $2 \mathrm{ml}$ of PBS. Finally, the cells were resuspended in $1 \mathrm{ml}$ PBS for immediate flow cytometric analysis.

Antigen profile [12] and monoclonal antibodies to determine CECs and CEPs are listed in tables 2 and 3. Flow cytometry data were analyzed by DIVA software (BD). In each analysis, 600,000 total cells were acquired. For each blood sample, isotype-matched (PE and FITC fluorochrome) controls were analyzed in order to set the appropriate regions. To minimize false positive events, the number of double positive events detected with the isotype controls was subtracted from the numbers of CD133+ or CD146+, respectively. Cells expressing a specific marker combination were reported as a percentage of the number of gate events (total events).

\section{Cytokines Analysis}

This study employed a commercially available premixed human 27-plex panel of cytokines (Bio-Plex Cytokine reagent kit, BIO-RAD Laboratories, Hercules, Calif., USA) including CCL12 [Chemokine (C-C motif) ligand 12], CXCL10 (C-X-C motif chemokine 10), eotaxin, FGF-Bas (basic fibroblast growth factor), G-CSF (granulocyte colony-stimulating factor), GM-CSF (granulocyte-macrophage colony-stimulating factor), IFNg (interferon gamma), IL-1b, IL-1ra (IL-1 receptor a), IL-2, IL-4, IL-5, IL-6, IL-7, IL-8, IL-10, IL-12, IL-13, IL-15, IL-17, MCP1 (monocyte chemotactic protein 1), MIP1a (macrophage inflammatory protein 1 alpha), MIP1b (macrophage inflammatory protein 1 beta), PDGFBB (human platelet-derived growth factor BB), RANTES (regulated upon activation of normal $\mathrm{T}$ cell expressed and secreted), TNFa (tumor necrosis factor alpha) and VEGF (vascular endothelial growth factor). This system consists of multiplex beads and detection antibodies designed to quantitate, in $\mathrm{pg} / \mathrm{ml}$, multiple cytokines in a single serum sample. All samples stored at $-80^{\circ} \mathrm{C}$ were then thawed at the same time, centrifuged at $1,000 \mathrm{~g}$ at $4^{\circ} \mathrm{C}$ for $10 \mathrm{~min}$ and used immediately for measurement. Samples were tested in duplicate for each time point (T0, T3, T28, T56 and T84) at $1 / 3$ dilution in species-specific Bio-Plex sample diluent, and blank values (background) were subtracted from all readings. Premixed lyophilized cytokine standards, premixed anticytokine conjugated beads, detection antibodies and streptavidin phycoerythrin were prepared according to manufacturer instructions. All assays were read on the Bio-Plex protein array system and data analysis was performed with the Bio-Plex-Manager software (BIORAD Laboratories, Hercules, Calif., USA).

\section{Statistical Analysis}

Data obtained from cytofluorimetric and cytokine analysis were evaluated using the Mann Whitney U-test for parametric independent variables using SPSS software (version 17.00, SPSS. Chicago, Ill., USA). All p values $\leq 0.05$ were considered significant.
Table 2. CEC and CEP antigen expression profile

\begin{tabular}{llllll}
\hline & Syto 16 & Anti-CD45 & Anti-CD31 & Anti-CD146 & Anti-CD133 \\
\hline CECs & + & - & + & + & - \\
CEPs & + & - & + & - & + \\
\hline
\end{tabular}

Table 3. Monoclonal antibodies and reagents used to determine CECs and CEPs

\begin{tabular}{|c|c|c|c|}
\hline Reagents & Markers & Quantity & $\begin{array}{l}\text { Company/ } \\
\text { fluorochrome }\end{array}$ \\
\hline Syto 16 & nuclear stain & Dil 1:10,000 & $\mathrm{BD} / \mathrm{FITC}$ \\
\hline 7-AAD & viability marker & $20 \mu \mathrm{l}$ & $\begin{array}{l}\text { BD/ } \\
\text { PerCPCy } 5.5\end{array}$ \\
\hline Anti-CD45 & $\begin{array}{l}\text { to exclude } \\
\text { hematopoietic cells }\end{array}$ & $5 \mu \mathrm{l}$ & $\mathrm{BD} / \mathrm{APC}$ \\
\hline Anti-CD133 & $\begin{array}{l}\text { a progenitor cell } \\
\text { marker }\end{array}$ & $10 \mu \mathrm{l}$ & $\begin{array}{l}\text { Miltenyi } \\
\text { Biotech/APC }\end{array}$ \\
\hline Anti-CD31 & endothelial cell marker & $5 \mu \mathrm{l}$ & BD/PECy7 \\
\hline Anti-CD146 & endothelial cell marker & $20 \mu \mathrm{l}$ & $\mathrm{BD} / \mathrm{PE}$ \\
\hline
\end{tabular}

Table 4. Patient characteristics

\begin{tabular}{llllr}
\hline $\begin{array}{l}\text { Patient } \\
\text { No. }\end{array}$ & Age & $\begin{array}{l}\text { Gleason } \\
\text { biopsy }\end{array}$ & TNM stage & $\begin{array}{l}\text { PSA } \\
\text { Tot-free }\end{array}$ \\
\hline 1 & 67 & $6(3+3)$ & pT1c pNx pM & $5.89-1.05$ \\
2 & 76 & $7(3+4)$ & pT2ac pN0 pM & $3.27-0.72$ \\
3 & 65 & $6(3+3)$ & pT2a pN0 pM & $1.93-0.42$ \\
4 & 70 & $6(3+3)$ & pT2a pN pM & $3.05-0.32$ \\
5 & 66 & $6(3+3)$ & pT2a pN0 pM & $7.84-1.24$ \\
6 & 70 & $6(3+3)$ & pT1c pN0 pMx & $9.52-2.11$ \\
7 & 56 & $6(3+3)$ & pT1c pN0 pMx & $9.50-0.63$ \\
8 & 77 & $7(3+4)$ & pT2a pN0 pM & $7.44-1.47$ \\
9 & 76 & $7(3+4)$ & pT2a pN1 pM & $9.3-0.58$ \\
10 & 78 & $7(3+4)$ & pT2a pN0 pM & $4.14-0.82$ \\
\end{tabular}

PSA $=$ Prostate-specific antigen; TNM $=$ Tumor/Lymph Nodes/Metastasis classification of malignant tumors.

\section{Results}

Patient Characteristics

Table 4 summarizes the characteristics of patients enrolled in the study.

\section{CEC and CEP Behavior under ZOL Treatment}

CEC and CEP levels were directly quantified by flow cytometry from the whole blood of the control subjects and the 10 enrolled patients. In healthy controls $(n=3)$, 
Table 5. Summary of CEC and CEP mean percentage ( \pm SD) and statistical analysis

\begin{tabular}{lllllll}
\hline Time points & CEC mean (\%) & SD & p value & CEP mean (\%) & SD & p value \\
\hline T0 & 0.001417 & 0.002506 & & 0.00314 & 0.002942 & \\
T3 & 0 & 0 & $\mathbf{0 . 0 1 4}$ & 0.001267 & 0.001231 & 0.230 \\
T28 & 0.00015 & 0.000173 & 0.083 & 0.0018 & 0.001061 & 0.173 \\
T56 & 0.0001 & 0.000141 & 0.156 & 0.0006 & 0.000187 & 0.063 \\
T84 & 0.000183 & 0.000325 & 0.190 & 0.000229 & 0.000243 & $\mathbf{0 . 0 0 9}$ \\
\hline
\end{tabular}

Figures in bold: $\mathrm{p} \leq 0.05$.

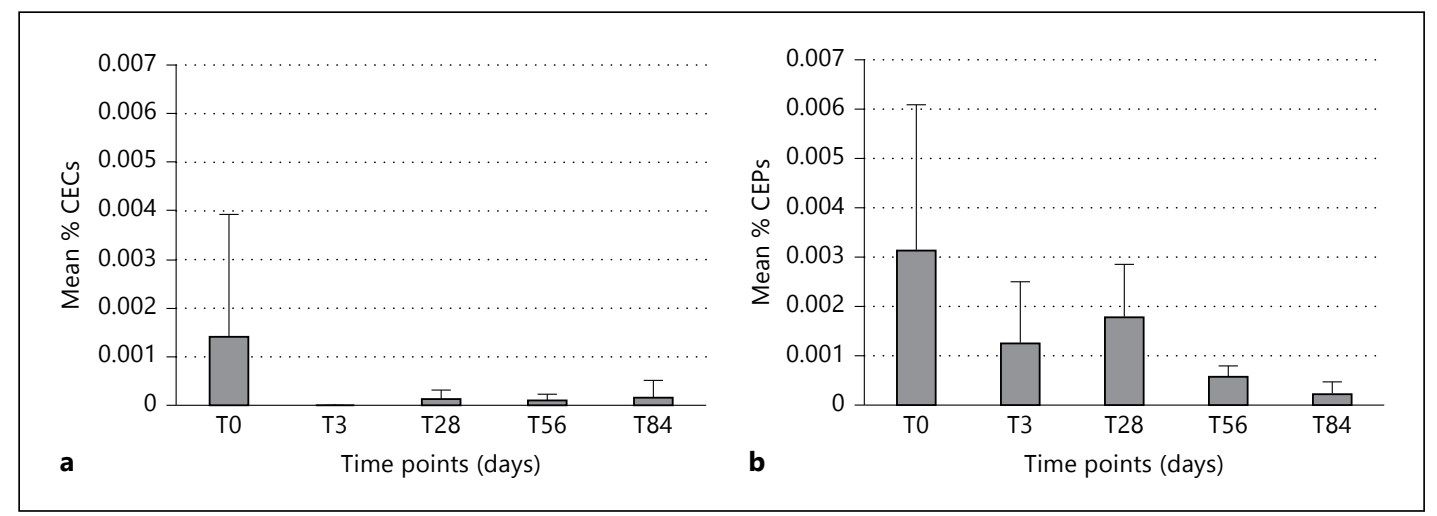

Fig. 1. Flow cytometric analysis of CECs and CEPs. Mean percentage ( \pm SD) of CECs $(\mathbf{a})$ and CEPs $(\mathbf{b})$ at different time points (T0, T3, T28, T56 and T84).

mean percentages of CECs and CEPs were 0 and $0.0002 \%$, respectively. In table 5 , the mean CEC and CEP percentages for the 10 patients evaluated at all time points are summarized.

The value of CECs decreased significantly at day 3 $(p=0.014)(a$ decrease of $100 \%)$, but this significance was not confirmed at the subsequent time points (fig. 1). A reduction of mean CEP percentage was observed at all time points compared to baseline (a decrease of 60, 43,81 and $93 \%$ at T3, T28, T56 and T84, respectively) (fig. 1). A representative example of the behavior of CEPs on flow cytometry is shown in figure 2 . The reduction of CEP count reached statistical significance at T84 $(\mathrm{p}=0.009)$.

\section{Cytokine Results}

In order to evaluate the effect of ZOL treatment on different soluble proteins and peptide modulation in our 10 patients, we simultaneously analyzed the serum levels of 27 cytokines for each of the time points (T0, T3, T28, T56 and T84).

Effect of ZOL on CECs and CEPs in Early Prostate Cancer
Detectable cytokine expression levels were obtained for only 20 cytokines; among these, only 13 showed a statistically significant change in serum concentration ( $\mathrm{p} \leq 0.05)$. We stratified these 13 cytokines into 3 groups: proinflammatory (IL-1b, IL-6, IL-8, IL-12, IFNg and TNFa), Th2 (IL1ra, l'IL-4, l'IL-5 and IL-10) and others (IL-7, IL-9 and RANTES). Our data demonstrated a statistically significant increase in serum concentration of the proinflammatory, Th2 and other cytokines, except for RANTES which demonstrated an opposite response to ZOL at different time points. Of relevance, all cytokines displayed a statistically significant modification of serum concentration at T84. Results were analyzed comparing cytokine levels at different time points with the baseline levels (data not shown).

\section{Discussion}

The aim of this study was to evaluate the antiangiogenic properties of ZOL treatment in patients with localized prostate cancer candidated to radical prostatectomy,

Oncology 2013;85:342-347 345 


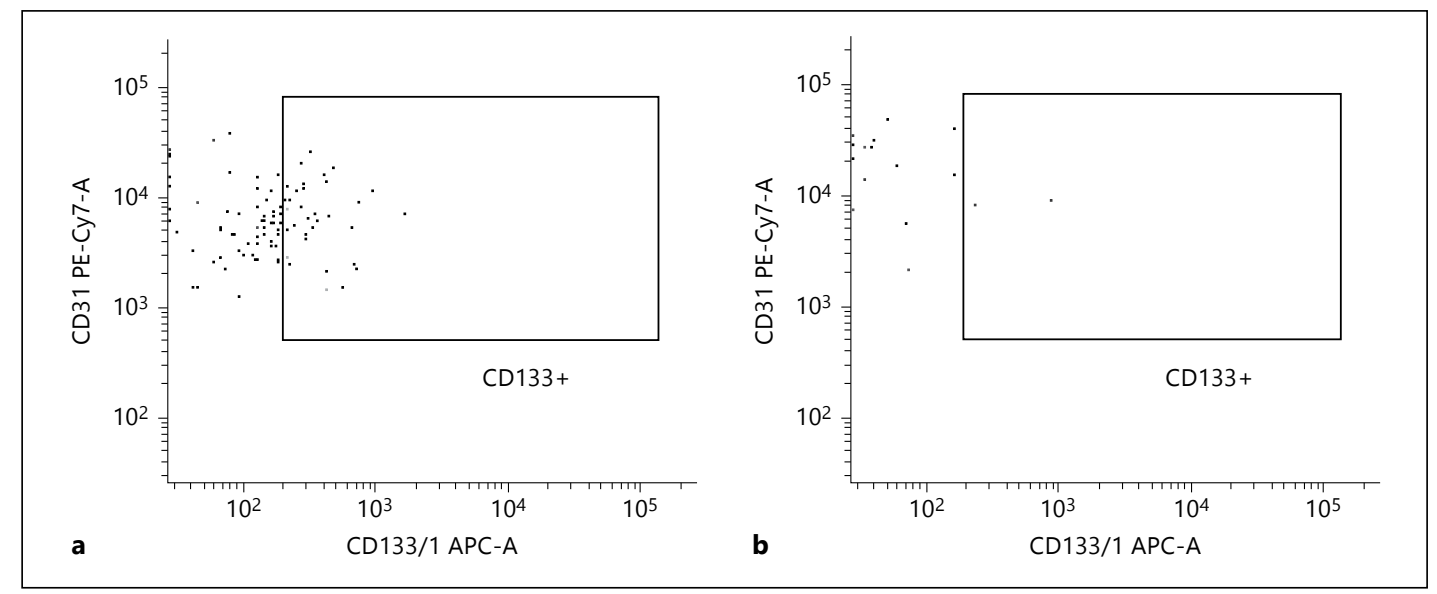

Fig. 2. Flow cytometric detection of CEPs in peripheral blood at T0 (a) and T84 (b). A significant reduction of CEPs can be observed at T84 compared to T0.

by analyzing early changes in new angiogenic biomarkers such as CECs and CEPs.

Moreover, the possibility of carrying out a watchful waiting approach delivers a unique opportunity to evaluate new angiogenetic markers useful to monitor $\mathrm{ZOL}$ anticancer efficacy, thereby minimizing the impact of potential confounding factors deriving from combination therapy.

Endothelial cells detected in peripheral blood may reflect the angiogenetic process and their modulation could be used as a surrogate marker of the antiangiogenetic therapy efficacy [9]. We were able to identify two different endothelial cell subsets named CECs and CEPs. CECs, characterized by CD146 expression, are cells arising from vessel wall turnover; CEPs, however, are bone marrow-originating cells expressing the surface antigen $\mathrm{CD} 133$, gradually lost during maturation. Moreover, compared to CEPs, CECs are differentiated cells with a reduced proliferative capacity [9]. Recently, it has been demonstrated that the endothelial precursors, mobilized in response to signals generated by the underlying neoplasm, contribute to vasculogenesis $[13,14]$. CEPs are known to form the premetastatic niche, essential in the earliest step to tumor angiogenesis [15], and it has been shown that targeting CEPs inhibits the development of macrometastases from micrometastases in mouse lung metastatic models [16]. Very preliminary data emerging from our study showed a statistically significant decrease in CEP number following treatment with ZOL. Although their number was characterized by a steady downward trend, the significance was achieved at the last time point
(T84). After administration, ZOL localizes and binds to bone matrix with high affinity, increasing its concentration within the bone. This cumulative effect due to multiple infusions could justify the direct action of ZOL against CEPs which are typically localized in the bone marrow where ZOL interferes with CEP proliferation/ mobilization. Indeed, some in vitro evidence have recently demonstrated that ZOL exerts both a significant inhibitory effect on proliferation and triggers apoptosis of cultured human endothelial cells, inducing apoptosis only at high concentrations $[11,17]$. The results concerning CECs showed a statistically significant decrease in their number only at T3. We hypothesize that the short ZOL half-life in circulation may explain the transient effect on CEC number. In fact, these cells are directly derived from vessels subjected to remodelling processes in the circulatory system where ZOL plasma levels rise, reach a peak and then fall rapidly after systemic administration. Moreover, the result concerning the decreased levels of RANTES, known to be secreted by mesenchymal stem cells, could reflect, in our series, the biological activity of ZOL on these cells within bone marrow, leading to a reduction of their ability to produce chemotactic signals involved in cancer cell migration $[18,19]$.

As previously demonstrated, CEPs are recruited to participate in the formation of the premetastatic niche in the earliest step of the metastatic process [15]. Intriguingly, ZOL could act as a compound able to target, in an early phase of cancer diffusion, the premetastatic niche onset through the inhibition of CEP mobilization and of mesenchymal stem cell function.
Santini et al. 
In addition to it being demonstrated that $\mathrm{ZOL}$ directly inhibits the growth of prostate cancer cells, the significant effects that this drug exerts on CECs/CEPs and on RANTES might also counteract the ability of cancer cells to form distant metastases. The preliminary results of our study demonstrate for the first time that ZOL could exert a new potential antiangiogenic effect in the early prostate cancer neoadjuvant setting through the modulation of CECs and CEPs. Further experiments and studies are needed to confirm and consolidate these results.

\section{Acknowledgement}

Reagents for this work were partly funded by Novartis.

\section{References}

1 Russell RG, Xia Z, Dunford JE, et al: Bisphosphonates: an update on mechanisms of action and how these relate to clinical efficacy. Ann NY Acad Sci 2007;1117:209-257.

2 Santini D, Galluzzo S, Vincenzi B, et al: New developments of aminobisphosphonates: the double face of Janus. Ann Oncol 2007;18: 164-167.

3 Clézardin P, Ebetino FH, Fournier PG: Bisphosphonates and cancer-induced bone disease: beyond their antiresorptive activity. Cancer Res 2005;65:4971-4974.

4 Santini D, Vincenzi B, Dicuonzo G, et al: Zoledronic acid induces significant and longlasting modifications of circulating angiogenic factors in cancer patients. Clin Cancer Res 2003;9:2893-2897.

5 Du R, Lu KV, Petritsch C, et al: HIF1alpha induces the recruitment of bone marrow-derived vascular modulatory cells to regulate tumor angiogenesis and invasion. Cancer Cell 2008;13:206-220.

6 Lyden D, Hattori K, Dias S, et al: Impaired recruitment of bone-marrow-derived endothelial and hematopoietic precursor cells blocks tumor angiogenesis and growth. Nat Med 2001;7:1194-1201.
7 Shaked Y, Ciarrocchi A, Franco M, et al: Therapy-induced acute recruitment of circulating endothelial progenitor cells to tumors. Science 2006;313:1785-1787.

8 Matsusaka S, Mishima Y, Suenaga M, et al: Circulating endothelial progenitors and CXCR4-positive circulating endothelial cells are predictive markers for bevacizumab. Cancer 2011;117:4026-4032.

9 Bertolini F, Shaked Y, Mancuso P, Kerbel RS: The multifaceted circulating endothelial cell in cancer: towards marker and target identification. Nat Rev Cancer 2006;6:835-845.

10 Blann AD, Woywodt A, Bertolini F, et al: Circulating endothelial cells, biomarker of vascular disease. Thromb Haemost 2005;93:228-235.

11 Yamada J, Tsuno NH, Kitayama J, et al: Antiangiogenic property of zoledronic acid by inhibition of endothelial progenitor cell differentiation. J Surg Res 2009;151:115-120.

12 Mancuso P, Antoniotti P, Quarna J, et al: Validation of a standardized method for enumerating circulating endothelial cells and progenitors: flow cytometry and molecular and ultrastructural analyses. Clin Cancer Res 2009; 15:267-273.

13 Carmeliet P, Luttun A: The emerging role of the bone marrow-derived stem cells in (therapeutic) angiogenesis. Thromb Haemost 2001; 86:289-297.
14 Rafii S, Lyden D, Benezra R, Hattori K, Heissig B: Vascular and haematopoietic stem cells: novel targets for anti-angiogenesis therapy? Nat Rev Cancer 2002;2:826-835.

15 Kaplan RN, Riba RD, Zacharoulis S, et al: VEGFR1-positive haematopoietic bone marrow progenitors initiate the pre-metastatic niche. Nature 2005;438:820-827.

16 Gao D, Nolan DJ, Mellick AS, Bambino K, McDonnell K, Mittal V: Endothelial progenitor cells control the angiogenic switch in mouse lung metastasis. Science 2008;319:195-198.

17 Stresing V, Fournier PG, Bellahcène A, et al: Nitrogen-containing bisphosphonates can inhibit angiogenesis in vivo without the involvement of farnesyl pyrophosphate synthase. Bone 2011;48:259-266.

18 Gallo M, De Luca A, Lamura L, Normanno N: Zoledronic acid blocks the interaction between mesenchymal stem cells and breast cancer cells: implications for adjuvant therapy of breast cancer. Ann Oncol 2012;23:597604.

19 Insalaco L, Di Gaudio F, Terrasi M, et al: Analysis of molecular mechanisms and anti-tumoural effects of zoledronic acid in breast cancer cells. J Cell Mol Med 2012;16:2186-2195. 